\title{
LINKS BETWEEN JET INSTABILITIES, RADIATION AND PROPAGATION IN ASTROPHYSICS*
}

\author{
Gregory Benford \\ Department of Physics \\ University of California \\ Irvine, California 92717
}

ABSTRACT

At the head of a jet the confining medium of plasma frequency $\nu$ is compressed, so that streaming instabilities between relgtivistic electrons and this plasma produce waves at $v^{\prime}>v^{\prime}$. Considerable power can be lodged in these electrostalic waves, and conversion to electromagnetic waves allows them to propagate far beyond the jet. ${ }_{2}$ Emission at $\nu \approx \nu$ ' or Compton boosted radiation at $\nu \lesssim \gamma^{2} \nu$ ' yields a cone $\mathrm{p}$ of radiation of angle $\sim 1 / \gamma$, which ill $\sim$ minates the region directly in front of the jet. This emission is not absorbed by the surrounding plasma unless a cloud blocks the jet. Absorption in a cloud can lead to tunneling through large clouds, or propelling of smaller clouds out of the jet path. In this fashion jets may clear their way through an inhomogeneous medium, avoiding lateral disturbances and preheating their path.

\section{INTRODUCTION}

We often assume that the propagation of astrophysical jets is a matter of macroscopic physics -- ram pressure balance, Kelvin-Helmholtz instabilities and the like. Microscopic process are responsible for reacceleration -and thus the radio emission whereby we see the jets -- but seem to have little impact on large scales.

This need not be so. One of the primary difficulties in understanding jets is how they can pass relatively unperturbed through a medium which must contain irregularities - clouds of dense gas, random gradients, and perhaps large magnetic fields. Dense clouds particularly seem to be a problem for good jet propagation, since a cloud can deflect a jet abruptly, driving instabilities on a scale of 
the jet radius. Since it seems likely that clouds are common in intergactic space, propagation along a straight line for megaparsecs, as in Cen A, requires an explanation. Similar difficulties apply to small jets such as SS433 and perhaps the Crab jet, since their environments are certainly highly inhomogeneous.

The primary assumption made in studying such effects is that the beam can deliver energy to the surrounding gas only at the "working surface" -- a region where shocks and rapid energy deposition achieve the tunnel boring, producing synchrotron hot spots. Here I propose a simple mechanism whereby beams can affect the plasma beyond the working surface, perhaps many beam radii ahead. This mechanism selects high density irregularities for energy deposition, and thus acts as an "intelligent" preparer of the downstram environment.

\section{BASIC PICTURE}

The jet of density $n_{j}$ and speed $v_{j}$ collides with a dense surrounding gas (density $n_{g}$ ) at the working surface, compressing this gas to a higher density $n_{g}^{\prime}$. Ahead of the jet, a dense cloud of density $n_{c}$ waits. If the jet could not affect the cloud until it struck, deflections could be considerable. (Fig. 1)

However, the beam is a source of electromagnetic radiation. Aside from synchrotron emission, which is of high frequency, there can be copious collective emission at or above the local plasma frequency, $\nu_{\mathrm{p}}\left(\mathrm{n}_{\mathrm{g}}{ }^{\prime}\right) \geqslant 100 \mathrm{~Hz}$. The
key features of this mechanismare:

1. The jet compresses the gas at the working surface, so emission is at $\left.\nu\left(n{ }^{\prime}\right)>\nu_{(n}\right)$. Since collective processes in the homogeneous $\mathrm{gas}^{g}\left(\mathrm{n}_{\mathrm{g}}\right)$ absorb only at $\nu<\nu\left(n_{g}\right)$, the radiation propagates freely. It will be absorbed ${ }^{g}$ only by clouds denser than $\mathrm{n}_{\mathrm{g}}$ '.

2. Emission is intense, if our knowledge of Type III solar bursts and of laboratory beam-plasma experiments is any guide.

3. Emission is concentrated into a cone of opening angle $\Omega=1 / \gamma+\phi$, where $\gamma$ is the Lorentz factor of the beam electrons and $\phi$ is the average opening angle of the beam electron trajectories in the emitting area. This serves to concentrate the power into the region downstream where the beam must clear a path. 


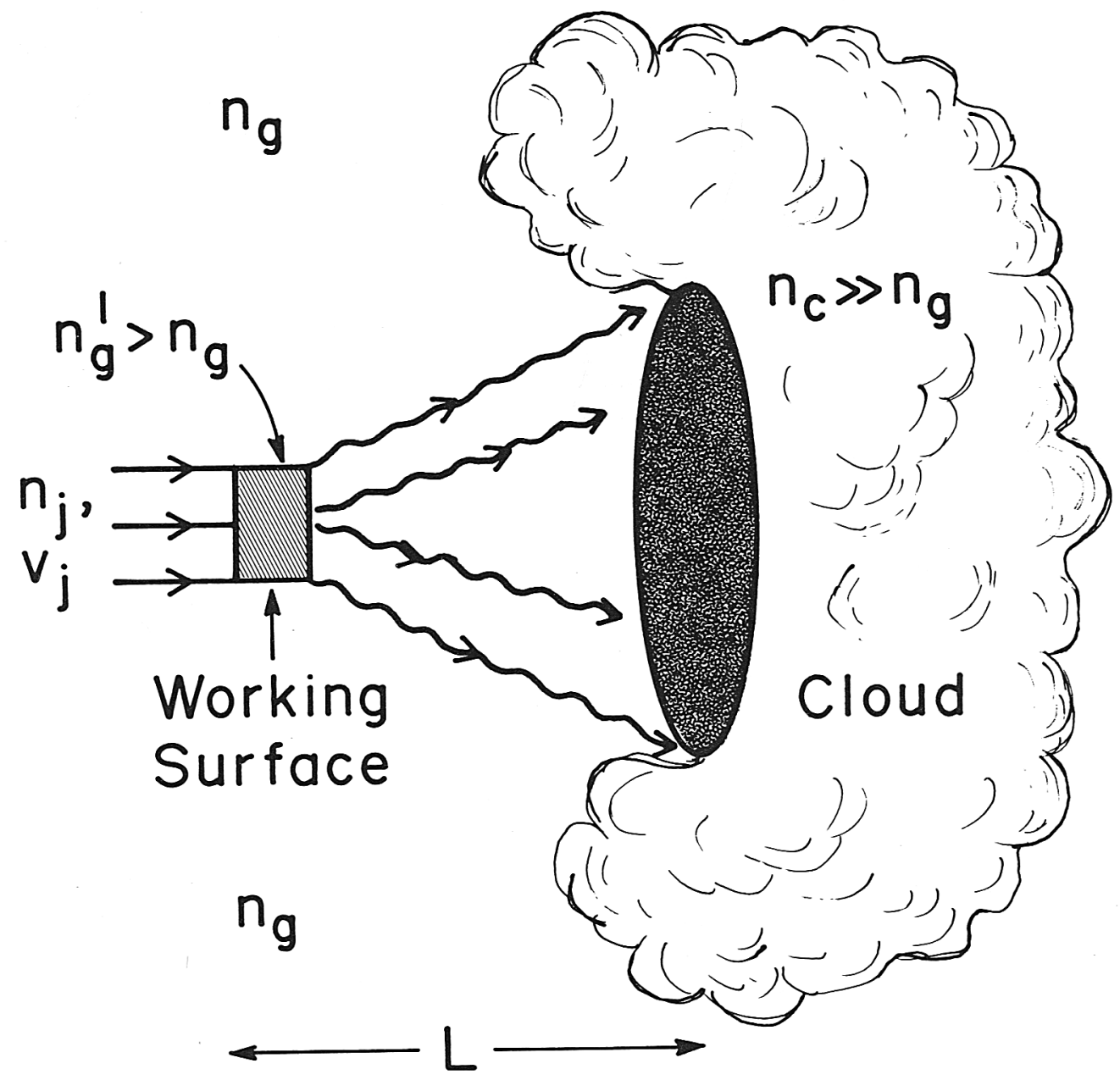

Fig. 1. A jet compresses the surrounding plasma to a higher density, $\mathrm{n}^{\prime}$ at the working surface. Electrostatic waves produced by beam-plasma instabilities there lead to electromagnetic emission. A cloud is dispersed by this radiation before the jet arrives. 
4. Absorption of the radiation in the denser cloud will heat this cloud, causing it to expand, reducing the density. When $n_{c}$ drops to $n_{g}^{\prime}$, the photons pass through, so they can do further tunneling downstream. Thus energy is delivered to a cloud only so long as it acts as a jarring barrier to the (presumably) smooth tunneling going on.

The plasma waves are produced at the working surface by beam-plasma instability. Presumably these are among the processes which eventually clear the beam path of high density plasma. Nonlinear processes then convert these electrostatic waves to electfquagnetic photons of very low frequency, $\nu{ }^{\prime}\left(n^{\prime}\right) \approx 100 n^{\prime} \mathrm{Hz}^{\prime}$. These photons are unobservable ${ }^{\text {pbut }}$ carry a high flux of energy.

\section{COLLECTIVE EMISSION PROCESSES}

Decades-long study of the Type III solar radio radiation has yielded detailed scenarios for electromagnetic emission (Smith, 1970, and references therein). Recent experiments have verified some aspects of the weak turbulence emission scheme, both for relativistic beams (Benford, et al, 1980) and nonrelativistic cases (Cheung et a 1, 1982; Whelan \& Stenzel, 1981). Such processes can be very efficient because there is spatial amplification of the electromagnetic waves as they propagate through a background of large amplitude electrostatic waves (driven by beamplasma instability). We shall use the theoretical model of weak turbulence emission at $\nu$ and apply it to the working surface, where a weak beam pehetrates a dense plasma.

The volume emissitivity of the emission (Benford $\&$ Smith, 1982) for plasma of temperature $T$ in eV is

$$
J=6 \times 10^{-16} \frac{\mathrm{Tk}_{\mathrm{m}}{ }^{2}{ }_{\mathrm{p}}{ }^{2}\left(\mathrm{n}_{\mathrm{g}}{ }^{\prime}\right)}{\mathrm{n}_{\mathrm{g}}{ }^{\prime}} \mathrm{W}
$$

and the power emitted by the beam at $\nu\left(n{ }^{\prime}\right)$ is $p=V J e^{\mu x}$ with $V$ the volume, $x$ the characteristil mean distance an electromagnetic wave travels while being amplified, and

$$
\mu=\frac{6.610^{-34} \mathrm{~W}_{\mathrm{p}^{2}}\left(\mathrm{n}_{\mathrm{g}}{ }^{\prime}\right)}{\mathrm{T}^{3 / 2} \mathrm{k}_{\mathrm{p}} \mathrm{n}_{\mathrm{g}}{ }^{\prime}}
$$

is the amplification factor. It depends on $W$, the energy density of the plasma waves, which in turn depends on the nonlinear saturation mechanism. We shall suppose weak turbulence processes saturate the electrostatic waves at a 
Low amplitude given by (Papadopoulos, 1975)

$$
w \approx n_{j} \gamma m c^{2} \frac{\delta}{\nu_{p}\left(n_{g}{ }^{\prime}\right)}
$$

where $\delta$ is the linear wave growth rate for a warm beam which has a large spread in emission angle,

$$
\delta \approx \nu_{p}\left(\mathrm{n}_{\mathrm{g}}{ }^{\prime}\right) n \frac{1}{\gamma \phi^{2}}
$$

Here $\phi$ is the angular spread of the beam electrons and $n=n_{j} / n_{g}$. The typical wavenumber of the turbulence, $k_{p}$, is taken as $\left.{ }^{\circ} \nu(n)^{\prime}\right) / c$. The largest $k$ present, $k_{m}$ is $t a k e n$ as the same Porger.

In this model, emission at $2 \nu$ is much less powerful, and cannot be amplified by the relalively slow plasma waves. Thus we neglect such radiation.

In terms of astrophysical parameters (cgs units)

$$
\begin{aligned}
& \mathrm{W} \cong 10^{-9} n^{2} \phi^{-2} \mathrm{n}_{-4} \\
& \mathrm{~J} \cong 2 \times 10^{-32} \mathrm{~T} \mathrm{n}_{-4}^{2}(n / \phi)^{2} \\
& \mu=6 \times 10^{4} \mathrm{n}_{-4}^{1 / 2} \mathrm{~T}^{-3 / 2\left(n_{-3} / \phi_{-1}\right)^{2}} \\
& \mathrm{P}=2 \times 10^{29} \mathrm{~T} \mathrm{n}_{-4}^{2}\left(n_{-3} / \phi_{-1}\right)^{2} \mathrm{R}^{3}(\mathrm{y} / \mathrm{R}) \mathrm{e}^{\mu \mathrm{x}}
\end{aligned}
$$

Here we write $n_{-4}=n_{g} / 10^{-4} \mathrm{~cm}^{-3}, \mathrm{~T}$ the plasma temperature in eV., R the beam ragius in kpc, n-3 the beam-plasma density ratio in units of $10^{-3}$, etc. The working surface has a thickness $y$, and we expect $y / R \sim 0.1$.

Since 5 spatial amplification occurs over an e-folding length $10^{5} \mathrm{~cm}$., we can expect a great increase in emission above the simple volume emissivity J. This is typical of Type III bursts, and also agrees with laboratory work, where the product $\mu x \sim 25$ is compatible with observed radiated power (Benford \& Smith, 1982). Such a short amplification length means that to disrupt amplification requires wave refraction on a scale much less than the typical wavelength of the beam-plasma instability, 
productive losses -- i.e., those which smooth out the beam path -- occur only to the sides. We shall content ourselves with : a naive estimate of the heating time, assuming that simple heating and expansion occur. A tunnel of width $R$ must push cloud matter within a distance $R_{c}$, the cloud radius, out of the way to expand, so that heating times are lengthened by the area factor $\left(R_{c} / R\right)^{2}$. Cases in which the cloud is not much larger than the jet radius clearly will be less "loaded", since the heated matter can escape into lower density regions. When the cloud is smaller than the jet radius, it can even be shoved to the side by a rocket effect.

The beam compresses the surrounding gas to density $\mathrm{n}^{\prime}$ over a distance $L^{\prime}$ and radially over a scale $R^{\prime}$. This pressure differential is relieved by sound waves in the cool plasma of speed U. The amplification of density is roughly

$$
\mathrm{n}_{g}{ }^{\prime / \mathrm{n}_{\mathrm{g}}} \approx 1+\frac{\mathrm{U}}{\mathrm{v}_{\mathrm{j}}} \frac{\mathrm{L}^{\prime}}{\mathrm{R}^{\top}} n^{-1 / 2}
$$

If we estimate $U / v \sim 0.1, L^{\prime} / R^{\prime} \sim 1$ and $n \sim 10^{-3}$, this yields density contrasts of order unity. This insures that local emission at $\nu^{\prime}\left(n^{\prime}{ }^{\prime}\right)$ will propagate freely at very low frequencies, $\nu_{p}\left(n_{g}{ }^{p}\right)^{g}{ }_{2} 100 \mathrm{~Hz}$ until encountering a cloud.

Using (2); a beam can heat a cloud until $n=n$ if it begins irradiation at a distance $L_{0}$ and the process geases at $\mathrm{L}_{\mathrm{f}}$, if

$$
L_{O}=\frac{L^{*} L_{f}}{L^{*}-L_{f}}
$$

where $L^{*}=\frac{R^{2}}{c t_{h}}\left(\frac{R}{R_{c}}\right)^{2}(k p c)^{2}$,

$t_{h}=\left(\frac{n_{c}}{n_{g}}\right)\left(\frac{T}{T}\right)\left(\frac{\phi}{n_{-3}}\right)^{2}\left(\frac{z}{y}\right) 10^{21} e^{-\mu X}$ rec

Here $z$ is the length of the heated zone, with radius of 1 $\mathrm{kpc}$ and $R_{c}$ is the cloud radius. Clearly, th achieve heating in the closing time $t_{c}=310^{-2}\left(\mathrm{c} / \mathrm{v}_{\mathrm{b}}\right) n_{-3}$ sec, we need $\mu X \approx 16$ when $R=R$, which is less than the amplification typically invoked for laboratory experiments and Type III bursts (Benford \& Smith, 1982).

With this amplification, $L^{*} \sim \mathrm{R}^{2} / 10 \mathrm{kpc}$ and $\mathrm{L}_{\mathrm{O}}<\mathrm{R} \sim$ kpc. The problem is complicated if $L \sim R \sim k p c$, but the range of $L_{0} \lesssim i 0 \mathrm{kpc}$ assures that the effect can operate if 
the beam has $\sim 10^{6}$ yr to heat the cloud. For these estjmates we have taken $\left(\mathrm{n}_{\mathrm{c}} / \mathrm{n}_{\mathrm{g}}\right) \sim 10,(\mathrm{~T} / \mathrm{T}) \sim 0.1$, z $\phi^{2} / y \sim 1$. Allowing for ${ }^{2}$ lateral inhibition of expansion through the factor ( R/R $)^{2}$ lengthens the true heating time, yet there seems ample freedom in the value of $\mu L$ to accomodate this movement of gas.

In terms of efficiency, we can express the requirement that a jet heat and disperse a cloud of depth d in terms of the ratio of $P_{r}$, power radiated at $\sim \nu_{p}$, to $P_{j}$, the jet power at the working surface. For clobd dispersal,

$$
\frac{P_{r}}{P_{j}}>\varepsilon_{-1}\left(\frac{n_{c}^{T} c}{n_{g}^{T}}\right)_{1} n_{-3}^{1 / 2}\left(\frac{d}{L}\right)_{-2}\left(1+\frac{L}{\gamma R}\right)^{2} 0.03 \%
$$

The efficiency of jet boring through the ambient gas, $n_{g}$, is $\varepsilon$ in units of 0.1 , and the $1+L / \gamma R$ arises from the $\gamma-c$ gne geometry. Thus for plausible clouds, d/L 0.01 , a very mild efficiency $\sim 0.03 \%$ is needed. The U.C.I. beam-plasma experiments with $\gamma=3$ have efficiencies of this order, as do many other experiments in the nonrelativistic regime. Certainly thin clouds, (d/L) $-2<1$, are easy to disperse. To see if the effect is dominant for thick clouds, $\mathrm{d} / \mathrm{L} \sim 0.1$, requires detailed knowledge of jet conditions beyond our current ability.

This qualitative success implies that electromagnetic tunneling may be an effective agent. The general picture should work for jets of any size. The self-tuning feature of this model arises from the unique nature of plasma waves. Unlike synchrotron radiation, plasma emission is absorbed in very small distances if $\nu(n)>v\left(n{ }^{\prime}\right)$. Thus its effects are local and immediate. Phe emis $p^{c}$. T is of unobservably low frequency, except perhaps in dense, ss433-1ike environments. Indeed, electromagnetic tunneling may be crucial in the initial setting-up of jets in dense environments, since it could clear a zone in which a nozzle can then be self-consistently made, using beam self-magnetic fields and sidewise external gas pressure.

It seems probable that pre-heating of small clouds can increase the confining pressure around the beam at later times, aiding stability. Magnetic fields in the working surface or in the clouds will have little effect on plasmon emission and subsequent radiation; this appears to be true of laboratory experiments. Thus we expect microscopic collective emission processes can contribute to macroscopic behavior of jets on all astrophysical scales.

*Work supported by AFOSR Grant No. 82-0233 


\section{REFERENCES}

Benford, G., D.F. Smith, Phys. Fluids 25, 1450 (1982). Benford, G., D. Tzach, K. Kato, and D. Smith, Phys. Rev. Lett. 45, $1182(1980)$.

Cheung, P.Y., A. Y. Wong, C.B. Darrow, and S.J. Qian, Phys. Rev. Lett. 48, 1348 (1982).

Kato, K.G., G. Benford, D. Tzach, Phys. Rev. Lett. 50, 1587 $(1983)$.

Papadopoulos, K., Phys. Fluids 18, 1769 (1975).

Smith, D.F., Adv. Astron. Astrophys. 7, 147 (1970).

Whelan, D.A. and R.L. Stenzel, Phys. Rev. Lett. 47, 95 (1981).

\section{DISCUSSION}

Spanglex: Given the Lorentz factors of electrons in extended extragalactic sources, and best estimates of the local plasma frequency, your Compton-boosted radiation should be in the observable radio range. Does the absence of spectral "turn-ups" at low frequencies place constraints on your model?

Benford: This process occurs only in a small working surface region, so not much power at very high frequencies comes out. This can easily be swamped by ordinary synchrotron emission from the surrounding hot spot. Also, it is beamed along the jet, not usually toward us. I expect the many more electrons at low $\gamma$, giving $\sim 100 \mathrm{~Hz}$ photons, do most of the cloud heating. A power law electron spectrum folded into eq. (1) yields this conclusion.

Hardee: A very ingenious idea. You solved the problem of heating the cloud, but you created another problem; the acceleration of particles

Benford: I assume reacceleration occurs throughout the jet, as the synchrotron radiation testifies. This occurs independently of the plasma instabilities at the working surface. Indeed, all the particle acceleration and electromagnetic emission comes from jet kinetic energy, through various Alfvén or plasmon waves.

Vasyliunas: Shouldn't the beaming be controlled by the $\gamma$ of the jet motion and not of the realtivistic electrons (which should be distributed more or less isotropically in the frame of the jet), so that there is no pronounced beaming unless the jet itself moves at relativistic speeds?

Benford: Electrons in the working surface are probably quite anisotropic. Collective emission occurs only where beam-plasma instability drives the plasmon spectrum. By the time electrons turn to the side they may well have left the region of beam-plasma instability; then they join the cocoon. I expect $\gamma \lesssim 10$ electrons do most of the emissiol anyway, since there are many more of them. Even if you are right, the $\gamma$-cone effect is not essential to making the power requirement work. 